eISSN: 2659-6482

DOI: https://doi.org/10.14201/pmrt.26447

\title{
EDUCACIÓN MUSICAL SUPERIOR Y PRÁCTICAS INSTRUMENTALES CONJUNTAS ENTIEMPOS DE COVID-19
}

Retos en los procesos de enseñanza-aprendizaje y nuevas propuestas basadas en la educación online

\section{Musical Higher Education and Collective Instrumental Practices in Times of COVID-19}

Challenges in Teaching-Learning Processes and New Proposals Based on Online Education

Sara PÉREZ BORRAJO

(Universidade da Coruña)

sara.pborrajo@udc.es

Aarón PÉREZ-BORRAJO @ https://orcid.org/0000-0002-4049-4856

(Universidad de Salamanca)

aaronborrajo@usal.es

RESUMEN: Esta investigación tiene como objetivo principal estudiar el impacto de la pandemia ocasionada por el COVID-19 en la educación musical superior. De esta forma, se analizan los procesos de enseñanza-aprendizaje y, concretamente, la modalidad de educación online adoptada en este ámbito durante este período. Asimismo, se profundiza en la implantación de nuevas perspectivas pedagógicas motivadas por este contexto sociosanitario, con un interés específico a la hora de abordar las prácticas instrumentales conjuntas. La situación de emergencia y de crisis ocasionada por el coronavirus necesita de una acción educativa y social que, en este caso, sea capaz de asumir y de responder ante la obligación de generar un proceso educativo que incida en una práctica instrumental conjunta de calidad en los centros 
SARA PÉREZ BORRAJO Y AARÓN PÉREZ-BORRAJO

EDUCACIÓN MUSICAL SUPERIOR Y PRÁCTICAS INSTRUMENTALES CONJUNTAS EN TIEMPOS DE COVID-19

musicales de enseñanza superior. Para ello, desarrollamos y proponemos un nuevo modelo metodológico que, respetando las limitaciones suscitadas por este contexto, tiene presentes las necesidades, inquietudes y características individuales del alumnado que cursa este tipo de asignaturas.

Palabras clave: educación musical; educación online; enseñanza superior; COVID-19.

ABSTRACT: The main objective of this research is to study the impact of the COVID-19 pandemic on higher music education. In this way, it analyses the teaching-learning processes and, specifically, the online education modality adopted in this field during this period. It also explores the implementation of new pedagogical perspectives motivated by this socio-sanitary context, with a specific interest in the approach to joint instrumental practices. The emergency and crisis situation caused by the coronavirus requires educational and social action that, in this case, is capable of assuming and responding to the obligation to generate an educational process that has an impact on quality joint instrumental practice in higher education music centres. To this end, we develop and propose a new methodological model which, while respecting the limitations raised by this context, takes into account the needs, concerns and individual characteristics of students taking this type of subject.

Keywords: music education; higher education; joint instrumental practices; COVID-19.

\section{INTRODUCCIÓN}

La enfermedad del COVID-19, causada por la infección del coronavirus SARS-CoV-2, fue descubierta a finales del 2019 en la ciudad china de Wuhan, capital y epicentro demográfico de la provincia de Hubei. Debido a su rápida difusión y propagación, supuso un enorme desafío social de carácter mundial que afectó a múltiples áreas. En este sentido, uno de los ámbitos más perjudicados fue el educativo. Partiendo de los datos proporcionados por la Unesco ${ }^{1}$, más de 127 millones de estudiantes de todos los niveles han vivido y experimentado una

1 Esta información se encuentra disponible en la sección «Interrupción y Respuesta Educativa» de la Unesco, desde donde se puede seguir el impacto del COVID-19 en la educación, visualizando su evolución a lo largo del tiempo: https://es.unesco.org/covid19/educationresponse [Última consulta: 10 de septiembre de 2021]. 
SARA PÉREZ BORRAJO Y AARÓN PÉREZ-BORRAJO

EDUCACIÓN MUSICAL SUPERIOR Y PRÁCTICAS INSTRUMENTALES CONJUNTAS EN TIEMPOS DE COVID-19

situación de desigualdad durante su adaptación a la «nueva normalidad». Igualmente, según esta institución, en torno al 90 \% de la población estudiantil sufre o ha sufrido una interrupción en su desarrollo formativo. El COVID-19 plantea grandes dificultades a la hora de configurar una formación presencial, obligando a la adopción de modificaciones en las estrategias de aprendizaje, en los métodos de enseñanza y en los procesos de evaluación.

Tras la aparición del SARS-CoV-2 en Wuhan, el Comité de Emergencia de la Organización Mundial de la Salud (OMS) se pronunció el 30 de enero del 2020 debido al riesgo de estar ante una emergencia sanitaria global ${ }^{2}$. En España, el 15 de marzo del 2020 se declaró el estado de alarma y un aislamiento domiciliario de, inicialmente, quince días ${ }^{3}$. El objetivo de este Decreto fue restringir la libertad de movimiento y de producción económica, prohibiendo las reuniones públicas y confinando a la mayor parte de la población. Únicamente se permitió el mantenimiento de aquellas actividades consideradas como esenciales e imprescindibles para asegurar el funcionamiento del sistema sanitario, del orden público y la provisión de productos de primera necesidad. Con esta drástica medida, el Gobierno de España perseguía alcanzar el pico de contagios, iniciando un proceso de desescalada en fases tras aplanar la curva.

El impacto del COVID-19 se encuentra a la altura de eventos tan dramáticos como la peste negra (1346-1352) o la gripe española (1918-1920). Según los datos proporcionados por el Coronavirus Resource Center de la John Hopkins University (Baltimore, Maryland), a fecha de 14 de julio de 2021 se estima que 188 millones de personas en todo el mundo han sido diagnosticadas como positivo por coronavirus, confirmándose el fallecimiento de más de 4 millones de ellas ${ }^{4}$. Se trata de un contexto trágico en lo sanitario, pero también en el desarrollo individual de la población mundial. La pandemia irrumpe en la vida cotidiana de las personas, pero no afecta por igual a todas, sino que presenta notables diferencias en función

2 Esta información se extrae de la «Declaración sobre la segunda reunión del Comité de Emergencias del Reglamento Sanitario Internacional (2005) acerca del brote del nuevo coronavirus (2019-nCoV)», Organización Mundial de la Salud. Disponible en: https://www. who.int/es/news/item/30-01-2020-statement-on-the-second-meeting-of-the-internationalhealth-regulations-(2005)-emergency-committee-regarding-the-outbreak-of-novel-coronavirus-(2019-ncov) [Última consulta: 1 de septiembre de 2021].

3 Real Decreto 463/2020, de 14 de marzo, por el que se declara el estado de alarma para la gestión de la situación de crisis sanitaria ocasionada por el COVID-19. Boletín Oficial del Estado, núm. 67, 14 de marzo de 2020, 25390-25400. Disponible en: https://www.boe.es/eli/ es/rd/2020/03/14/463

4 Sitio web del Coronavirus Resource Center de la John Hopkins University en el que se actualizan diariamente los datos de incidencia del COVID-19 en todo el mundo: https:// coronavirus.jhu.edu [Última consulta: 14 de julio de 2021]. 
SARA PÉREZ BORRAJO Y AARÓN PÉREZ-BORRAJO

EDUCACIÓN MUSICAL SUPERIOR Y PRÁCTICAS INSTRUMENTALES CONJUNTAS EN TIEMPOS DE COVID-19

de la posición que el sujeto ocupa dentro de la estructura socioeconómica, así como de la etapa vital en la que se encuentra.

Sin embargo, los períodos de crisis generan contextos proclives al cambio y a la adopción de nuevas estrategias. Asimismo, estos también ponen en evidencia la existencia de problemas que hasta el momento permanecían ocultos. Es decir, el COVID-19 y la crisis que trajo consigo no solo puso de manifiesto la fragilidad y el deterioro del ámbito sanitario en España, sino que además cuestionó la fortaleza del sistema educativo español, exponiendo las debilidades, carencias y desigualdades a las que se ven sometidos sus usuarios. En todos los niveles educativos, tanto docentes como familias y estudiantes se vieron obligados a responder de forma urgente ante un nuevo escenario determinado por la imposibilidad de llevar a cabo una educación presencial.

En este sentido, esta investigación se propone profundizar en las estrategias de enseñanza-aprendizaje no presenciales adoptadas en tiempos de pandemia, incidiendo en sus problemas, retos y posibilidades. Para ello, nos circunscribiremos al ámbito espacial de la Comunidad Autónoma de Galicia y a sus Conservatorios Superiores de Música ${ }^{5}$. Nuestro objeto de interés será la educación musical superior y, dentro de esta, las asignaturas instrumentales conjuntas. Ateniéndonos a lo establecido en el Decreto 171/2016 publicado el 12 de diciembre de 2016 en el Diario Oficial de Galicia ${ }^{6}$, en esta categoría se encuentran las siguientes materias: Música de cámara, Orquesta, Coro y Conjunto.

Música de cámara se imparte a lo largo de 8 cuatrimestres; cada uno de ellos consta de 3 créditos ECTS y de una carga lectiva de 90 minutos semanales. La asignatura de Orquesta oscila entre 4 y 8 cuatrimestres, con un total de 3,5 créditos ETCS por cuatrimestre y una carga lectiva de 180 minutos semanales. Coro, en función del itinerario y de la especialidad del alumno, se imparte entre 2 y 6 cuatrimestres, con una carga lectiva de 90 minutos semanales y 1 crédito ETCS por cuatrimestre. Conjunto oscila entre 2 y 8 cuatrimestres, con entre 2 y 3 créditos ETCS por cada uno de ellos y una carga lectiva de 90 minutos semanales. Con todo, es preciso señalar que, debido a la situación de pandemia, algunas de estas asignaturas se han reorganizado, dividiéndose en pequeños grupos para controlar

5 La Comunidad Autónoma de Galicia dispone de los siguientes centros de educación musical superior: el Conservatorio Superior de Música de Vigo (CSMV), el Conservatorio Superior de Música de A Coruña y el Centro Superior de Música de Galicia (CEMU Superior Galival Music).

6 Decreto 171/2016, do 24 de novembro, polo que se establece o Plan de estudos das ensinanzas artísticas superiores de Música, nas especialidades de Dirección e Produción e Xestión, e se amplían os anexos do plan de estudos correspondente á especialidade de Interpretación, establecidos no Decreto 163/2015, do 29 de outubro, mediante a incorporación de novos itinerarios. Diario Oficial de Galicia, núm. 235, 12 de decembro de 2016, 54101-54170. Disponible en: https://0151cc31-c939-46cb-8f09-f9239d6093df.filesusr.com/ugd/04f96b_ 2951ce8b598e4183a4065b1da4b96a3b.pdf 
SARA PÉREZ BORRAJO Y AARÓN PÉREZ-BORRAJO

EDUCACIÓN MUSICAL SUPERIOR Y PRÁCTICAS INSTRUMENTALES CONJUNTAS

EN TIEMPOS DE COVID-19

el aforo y mantener la distancia interpersonal recomendada. Concretamente, la asignatura de Orquesta se ha fraccionado en diferentes agrupaciones como orquesta de cuerda, orquesta barroca, ensemble de maderas, ensemble de metales y percusión, ensemble de clarinetes, banda, big band, etc.

\section{PROCESOS DE ENSEÑANZA-APRENDIZAJE Y EDUCACIÓN ONLINE}

Partiendo desde un enfoque estrictamente sociocultural, podemos entender el desarrollo ontológico del ser humano como un proceso que se encuentra determinado socialmente. Autores como L. Vygotsky (1972) consideran que el progreso y el perfeccionamiento cognitivo del sujeto resultan posibles gracias a su interacción con otros individuos con mayor conocimiento y experiencia. Es decir, el conjunto de relaciones interpersonales se establece y se articula a través de tareas compartidas. Un ejemplo podría ser la colaboración en una actividad orientada a la resolución de un problema concreto dentro de un marco cultural determinado y específico (Leontiev, 1981).

Por procesos de enseñanza-aprendizaje nos referimos a aquellas herramientas utilizadas por el docente con la finalidad de promover el desarrollo de las capacidades del alumno. Son procedimientos empleados, de manera reflexiva y flexible, para lograr un aprendizaje significativo. En estos términos, podemos definir el concepto de enseñanza como el cúmulo de acciones mediante las cuales se favorece la construcción de nuevos conocimientos de tipo formativo e informativo. Por lo tanto, el aprendizaje se constituye como la capacidad para obtener información, procesarla, interiorizarla y utilizarla para transformarla en conocimiento. Asimismo, hay que incidir en que este proceso no consiste únicamente en un aumento cuantitativo en el dominio de la información por parte del estudiante, sino también en la adquisición de habilidades para su empleo y aplicación desde una perspectiva cualitativa.

El sistema de enseñanza nunca debería limitarse a la transmisión directa de contenido entre el docente y el estudiante. La metodología y las estrategias de enseñanza-aprendizaje seleccionadas en función de la asignatura deberían promover una dinámica activa y participativa en la que el alumno pueda convertirse en el verdadero protagonista, actuando como agente activo a lo largo de su proceso de aprendizaje. Por ello, el objetivo del profesor consiste en promover capacidades como la autonomía y la autogestión, fomentando conductas y actitudes basadas en la creatividad, la motivación y el interés. En resumen, el docente cumple con un rol de orientador o guía, proporcionando soluciones concretas a problemas específicos, ampliando las capacidades de recepción-respuesta en el estudiante a lo largo de su desarrollo formativo. 
SARA PÉREZ BORRAJO Y AARÓN PÉREZ-BORRAJO

EDUCACIÓN MUSICAL SUPERIOR Y PRÁCTICAS INSTRUMENTALES CONJUNTAS EN TIEMPOS DE COVID-19

Sin embargo, el marco contextual de esta investigación se ve sometido a múltiples interferencias y modificaciones impuestas tras la aparición del COVID-19. Autores como Rujas y Feito (2021) destacan cómo esta pandemia mundial fue objeto de miles de estudios e investigaciones publicadas en diferentes plataformas socioeducativas en tan solo unos meses. El interés sobre los cambios y las alteraciones que el coronavirus trajo consigo provocó esta respuesta por parte del ámbito educativo y académico. El COVID-19 no solo irrumpe en una sociedad que ya estaba sometida a desigualdades socioeconómicas, culturales y digitales (Cabrera, 2020), sino que configura una realidad completamente diferente que merece ser analizada con detenimiento.

Durante el transcurso de la pandemia, la educación online se impuso como la primera opción y como una de las herramientas más interesantes para mantener activo, a pesar de sus limitaciones, el proceso de enseñanza-aprendizaje al que nos hemos referido anteriormente. Esta situación nos lleva a reflexionar sobre la necesidad de realizar un profundo cambio en el sistema educativo basado en las nuevas tecnologías de la información y de la comunicación (TIC) ${ }^{7}$. La consecución de esta revolución educativa, instituida a partir de la transferencia de los procedimientos didácticos a un plano digital y en las posibilidades de acceso del alumnado a dicha dimensión, es un requisito fundamental para adaptarse a una situación global inédita. Con todo, la presencialidad resulta insustituible. Las relaciones humanas que se establecen gracias a ella son más ricas, profundas y complejas. Las nuevas tecnologías limitan la comunicación, condicionando la espontaneidad y la percepción de sensaciones. Esta constricción comunicativa se manifiesta en el predominio de la frialdad y de la distancia frente a la empatía y al acompañamiento.

La diferencia principal entre la educación presencial y la educación a distancia -entre ellas la online- es el tipo de comunicación que se plantea entre los sujetos que integran el aula y el posible cambio en la relación que se establece entre el alumno y el docente. Por ello, entendiendo que este nuevo sistema de enseñanza necesita de estrategias y canales de comunicación alternativos, el desarrollo de las habilidades comunicativas se consolida como el gran desafío de la comunidad educativa (Rappoport, Rodríguez y Bressanello, 2020). Sin embargo, la realidad es que, en muchos de los casos en los que se implanta un modelo a distancia, el uso de la tecnología se limita únicamente a la transmisión de contenido, continuando

7 Más allá de esta dinámica transformadora, algunos investigadores apuestan por escenarios menos rupturistas y ambiciosos, persiguiendo una efectividad inmediata. Este es el caso de S. J. Daniel, quien considera que «This emergency is not the time to put into effect complex institutional plans for distance learning that were meant to be implemented over months or years [...]. Teachers should work with they know. Giving full attention to reassuring students is more important than trying to learn new pedagogy or technology on the fly» (2020: 93). 
SARA PÉREZ BORRAJO Y AARÓN PÉREZ-BORRAJO

EDUCACIÓN MUSICAL SUPERIOR Y PRÁCTICAS INSTRUMENTALES CONJUNTAS

EN TIEMPOS DE COVID-19

de este modo con los procedimientos empleados por la docencia presencial (García-Peñalvo y Corell, 2020: 85).

En definitiva, es en este contexto de crisis donde cabe destacar que la formación artística, y, específicamente, la educación musical, precisa de un profundo análisis y estudio para que su adaptación a la modalidad no presencial sea satisfactoria y eficaz. El coronavirus plantea enormes retos en el ámbito educativo, contribuyendo a aumentar una preocupación social ante el incremento de las desigualdades educativas ya existentes, amenazando con un bloqueo indefinido en los procesos de aprendizaje de consecuencias inimaginables. Sin embargo, tal y como venimos planteando, también sirvió para reforzar y legitimar la aplicación de nuevas estrategias procedimentales basadas en la transformación digital del sistema educativo. En este sentido, cabe preguntarse cuáles son y serán las dificultades con las que se encontrarán las reformas planteadas durante este escenario y si a partir de estas será posible avanzar hacia un sistema más equitativo e inclusivo en una fase de postpandemia.

\section{IMPACTO DEL COVID-19 EN LA EDUCACIÓN MUSICAL SUPERIOR: EDUCACIÓN ONLINE COMO ALTERNATIVA}

Atendiendo a nuestro objeto de investigación, procedemos a profundizar en las perspectivas y en las medidas adoptadas en el contexto espacio-temporal en el que este tiene lugar. Primeramente, debemos señalar que todavía existen pocas investigaciones dedicadas al análisis de los procesos de enseñanza-aprendizaje implantados en las instituciones musicales superiores tras la aparición del coronavirus. A pesar de que algunas de estas aportaciones valoran la posibilidad de establecer un sistema de educación online, tal y como puede ser el caso de Daubney y Fautley (2020), He (2020) o Kesendere, Sakir y Acar (2020), lo cierto es que los procesos formativos en este tipo de entidades todavía destacan por su conservadurismo en lo que respecta a la utilización de esta modalidad.

Sin embargo, estudios realizados con anterioridad al surgimiento del coronavirus examinan y exploran las posibilidades que ofrecen las nuevas propuestas de enseñanza y aprendizaje de música basadas en las TIC. Al respecto, destacamos las aportaciones de autores como Koutsoupidou (2013), Riley (2009), Orman y Whitaker (2010), Brändström et al. (2012), King et al. (2019), Cayari (2018), Brook y Upitis (2014), Waldron et al. (2017), Gammeren y Szram (2019) o Bayley y Waldron (2020). En cierto sentido, estas referencias pueden servirnos como punto de partida o marco teórico. Todas ellas subrayan y valoran las ventajas que plantea este tipo de educación no presencial. No obstante, para que este sistema educativo sea efectivo, es imprescindible la adquisición o la mejora de las habilidades TIC y de las competencias digitales tanto por parte de los docentes como del alumnado. 
SARA PÉREZ BORRAJO Y AARÓN PÉREZ-BORRAJO

EDUCACIÓN MUSICAL SUPERIOR Y PRÁCTICAS INSTRUMENTALES CONJUNTAS

EN TIEMPOS DE COVID-19

Con todo, al margen del desarrollo individual de estas, es necesaria la implicación de las instituciones y de las empresas y el establecimiento de estrategias más sólidas que permitan alcanzar una transformación digital real (García-Peñalvo, 2021).

Uno de los mayores obstáculos que surgen a la hora de reflexionar sobre la implantación de la educación online en las enseñanzas musicales superiores es la brecha o las barreras digitales ${ }^{8}$. El alumnado no dispone necesariamente de los mismos recursos, como podría ser el acceso a dispositivos electrónicos, el uso libre y/o exclusivo de estos, una conexión a internet de alta velocidad, etc. Además, las posibles diferencias en lo relativo a las características y a las propiedades de estos instrumentos electrónicos también pueden entorpecer la consecución de una participación equilibrada y abierta. Las posibilidades socioeconómicas de cada hogar y de cada familia no son las mismas; los espacios físicos desde los cuales los alumnos participan en el desarrollo de la asignatura tampoco. Por ello, uno de los objetivos primordiales de la educación online consiste en impedir que estas desigualdades logísticas y tecnológicas se trasladen tanto a la relación que el alumnado mantiene entre sí como a la que tiene respecto a la propia materia.

Este reto únicamente puede ser superado a partir de una firme respuesta institucional que asegure una participación en la educación online -también en el ámbito de las enseñanzas musicales superiores- en igualdad de condiciones. El hecho de que esta cuestión vaya más allá del objeto principal de esta investigación no impide que sea oportuno incidir en la necesidad de llevar a cabo un análisis sobre este problema9. Sin embargo, tampoco debemos olvidarnos de la existencia de dificultades compartidas entre docentes y alumnos en lo relativo al uso y empleo de las diferentes herramientas tecnológicas. En el caso del contexto que nos ocupa, lo más probable es que estos impedimentos hayan sido resueltos gracias al trabajo autónomo y autodidacta del sujeto, valiéndose de diversos recursos y de materiales complementarios disponibilizados digitalmente. A pesar de que desde la Administración se promueven iniciativas para solventar dichas deficiencias, estas no llegan por igual a todos los sectores que componen el sistema educativo.

Por otro lado, regresando a la realidad presencial o semipresencial adoptada en los Conservatorios Superiores de Música de Galicia durante este período, las primeras medidas implantadas para combatir la propagación del coronavirus

8 Autores como García-Peñalvo, Corell, Abella-García y Grande (2020) profundizan en este ámbito, diferenciando entre tres tipos de brecha. Si la primera de ellas tiene que ver con el acceso (aspecto logístico), la segunda se vincula a la propiedad y a las posibilidades de uso de los aparatos tecnológicos. Por último, la tercera estaría relacionada con las competencias del sujeto a la hora de desenvolverse de manera eficaz en dicha realidad digital.

9 En relación con este ámbito de estudio, debemos hacer referencia a las investigaciones de Lloyd (2020); Rodicio-García, Río-de-Deus, Mosquera-González y Penado Abilleira (2020), o Kuric, Calderón y Sanmartín (2021). 
SARA PÉREZ BORRAJO Y AARÓN PÉREZ-BORRAJO

EDUCACIÓN MUSICAL SUPERIOR Y PRÁCTICAS INSTRUMENTALES CONJUNTAS

EN TIEMPOS DE COVID-19

fueron tales como la estipulación de una distancia social mínima de 2 metros entre personas, el uso obligatorio de mascarilla, la colocación de un termómetro digital y de gel hidroalcohólico en cada una de las entradas y de las salidas de los centros, la apuesta por una organización y gestión más eficiente de los espacios, la desinfección de las aulas tras su uso, etc. ${ }^{10}$. No obstante, la realidad es que la puesta en práctica de muchas de estas normas no fue lo suficientemente efectiva, por lo que difícilmente se pudo garantizar una seguridad sanitaria entre los alumnos y los docentes.

Si llevar a cabo una presencialidad en las clases de instrumento fue complicado, incluso durante el curso lectivo 2020-2021, la gestión de una asignatura instrumental conjunta se convirtió en una tarea sumamente ardua. En un primer momento, agrupaciones de cámara, ensembles, bandas y orquestas optaron por una modalidad semipresencial mediante una división y rotación entre diferentes secciones instrumentales. No obstante, las cambiantes condiciones sociosanitarias, las circunstancias individuales y personales de cada alumno y docente y las diferentes medidas restrictivas tomadas por las instituciones en función del desarrollo de la pandemia imposibilitaron la estabilidad de este sistema.

Con todo, tal y como desarrollaremos posteriormente, debemos tener en cuenta que la enseñanza online de este tipo de asignaturas colectivas, al menos en lo que a la práctica instrumental se refiere, plantea grandes retos debido al factor de conectividad. Si el sonido no se capta y se reproduce al mismo tiempo en cada dispositivo, ¿cómo realizar una escucha e interpretación conjunta? De igual forma, la calidad de los recursos tecnológicos a disposición de alumnos y profesores es limitada, interfiriendo negativamente en el ámbito sonoro y visual de la intercomunicación, lo cual condiciona la acción pedagógica del docente y su intervención en la mejora de aspectos relativos al sonido, expresión, técnica, postura, interpretación, etc.

\section{PROPUESTA PARA UNA PRÁCTICA INSTRUMENTAL CONJUNTA ADECUADA A UN NUEVO CONTEXTO SOCIOSANITARIO}

La pregunta a partir de la cual se configura esta investigación es la siguiente: ¿Cuáles son las estrategias de enseñanza-aprendizaje más adecuadas para articular

10 Todos los centros de educación musical superior de Galicia disponen de protocolos ANTICOVID en los que se especifican las medidas a llevar a cabo para evitar la propagación del virus, la gestión de los espacios, los aforos máximos, los procedimientos y canales para informar de sintomatología, etc. Estos han sido creados y modificados a partir de las diferentes Ordes y Resolucións publicadas por la Xunta de Galicia en el Diario Oficial de Galicia desde el inicio de la pandemia. 
SARA PÉREZ BORRAJO Y AARÓN PÉREZ-BORRAJO

EDUCACIÓN MUSICAL SUPERIOR Y PRÁCTICAS INSTRUMENTALES CONJUNTAS EN TIEMPOS DE COVID-19

materias instrumentales conjuntas en centros musicales de enseñanza artística superior en tiempos de pandemia? Tras el análisis de las guías docentes de los Conservatorios Superiores de Música de Galicia correspondientes a las asignaturas instrumentales conjuntas del curso lectivo 2020-2021, identificadas y expuestas algunas de las dificultades y de las posibilidades de la educación online, y teniendo en consideración tanto las inquietudes del alumnado como las necesidades metodológicas y técnicas de los docentes, proponemos el siguiente proceso de enseñanza-aprendizaje adecuado a un nuevo contexto sociosanitario:

1. Reuniones previas entre los diferentes profesores que componen la asignatura -situación habitual en asignaturas instrumentales conjuntas- para organizar la materia, su distribución temporal y la asignación docente de los contenidos. La finalidad de este proceso consiste en establecer una hoja de ruta que permita al alumno saber cuándo, quién y qué se le va a impartir.

2. Reuniones entre el profesorado y el alumnado para exponer y explicar el sentido de la materia en el plan de estudios, las recomendaciones previas, los objetivos de la asignatura, los contenidos, las competencias a adquirir, las metodologías de trabajo, la previsión de técnicas docentes, los recursos complementarios y los procedimientos de evaluación. Estas sesiones también servirán para reflexionar conjuntamente sobre las posibilidades, los retos y las dificultades que entraña el establecer una modalidad de educación online. Para ello, se propone la utilización de plataformas como Microsoft Teams, Zoom, Skype (Microsoft), Webex o Google Meet. Al margen del aspecto económico, se debe priorizar el uso de aquellas que garanticen una mayor seguridad digital y privacidad de datos. En el caso de que fuera necesaria la instalación de programas para el acceso y participación, los estudiantes deberán ser avisados con antelación por correo electrónico, proporcionándose en el mismo las instrucciones necesarias para llevar a cabo tal proceso.

3. Contacto con cada alumno de manera individualizada para atender a sus necesidades logísticas específicas. Conocer la existencia de posibles carencias tecnológicas ayudará a solventar las barreras digitales y a garantizar su participación a través de la cesión de recursos o mediante una adaptación personalizada y justificada de las actividades a realizar. Es fundamental que esta comunicación sea exclusivamente entre el profesor y el alumno, a fin de mantener la privacidad de este último. 
SARA PÉREZ BORRAJO Y AARÓN PÉREZ-BORRAJO

EDUCACIÓN MUSICAL SUPERIOR Y PRÁCTICAS INSTRUMENTALES CONJUNTAS EN TIEMPOS DE COVID-19

4. Reunión colectiva en alguna de las plataformas anteriormente mencionadas para comentar el repertorio seleccionado para la asignatura. Aquí, el interés no radica en que el docente reflexione sobre las características musicales y extramusicales de las obras, sino en que esta tarea sea efectuada de forma conjunta. Es decir, promover un debate abierto sobre los aspectos contextuales, formales, armónicos, melódicos, rítmicos, sonoros, textuales, estilísticos, y las implicaciones de todos ellos a la hora de definir una praxis interpretativa adecuada.

5. Envío de grabaciones individuales de los pasajes más relevantes del repertorio seleccionado para cada instrumento. El objeto de estudio, el formato del archivo y la fecha de entrega serán acordados previamente con el profesor. Igualmente, este responderá con sus impresiones y comentarios (feedback).

6. Envío de grabaciones colectivas de pequeños grupos como duetos o tríos. Estas, pese a las barreras tecnológicas, permitirán trabajar en la escucha activa y en la coordinación conjunta de aquellos pasajes que más lo requieran. Esta actividad no solo se plantea entre plantillas compuestas por diferentes instrumentos, sino que también sirve para alumnos del mismo instrumento, permitiéndoles profundizar en ámbitos como la técnica, la expresividad o el sonido. Del mismo modo, estas grabaciones serán devueltas por parte del docente junto a indicaciones y correcciones.

7. Envío de grabaciones individuales sobre metrónomo del repertorio seleccionado. Tras la respuesta con las correcciones pertinentes por parte del profesor, el alumno deberá enviar una nueva grabación que permita evaluar la interiorización y el grado de aplicación de las indicaciones proporcionadas. Esta herramienta, con un período de tiempo definido entre envíos, permite observar la progresión -guiadadel estudiante. Para realizar esta tarea es necesario determinar previamente un programa de grabación, adjuntando las debidas instrucciones para su instalación y uso. Nuevamente, se tratará de sortear cualquier brecha digital que pudiera darse. Nuestra elección es Audacity, una aplicación informática libre que permite grabar y editar sonido, convertir e importar archivos, añadir efectos sonoros, incorporar plug-ins y editar pistas ${ }^{11}$. Este programa cuenta con una gran cantidad de plataformas y sistemas compatibles, por lo que se convierte en un recurso con grandes posibilidades.

11 Autores como Chao-Fernández, Vázquez-Sánchez y Felpeto-Guerrero (2020) profundizan en la incorporación y en la utilización de Audacity en la educación musical. 
SARA PÉREZ BORRAJO Y AARÓN PÉREZ-BORRAJO

EDUCACIÓN MUSICAL SUPERIOR Y PRÁCTICAS INSTRUMENTALES CONJUNTAS EN TIEMPOS DE COVID-19

8. Envío de grabaciones individuales sobre pista o audio del repertorio seleccionado. El material sonoro que actúa como base instrumental será proporcionado por el profesor; sin embargo, este no deberá aparecer de modo alguno en la tarea del alumno. El objetivo de esta actividad consiste en que el estudiante, inmerso en un modelo de enseñanza-aprendizaje progresivo y gradual, demuestre todos los conocimientos adquiridos a lo largo de dicho proceso formativo. Una vez más, estas grabaciones serán devueltas por parte del profesor junto a correcciones e indicaciones. La aplicación de las mismas se observará y se evaluará tras la entrega de la versión definitiva por parte del alumno.

9. El docente reunirá todas las grabaciones finales del repertorio seleccionado enviadas sobre audio. En colaboración con el alumnado, se superpondrán y se empastarán las pistas disponibles, generando una interpretación completa y colaborativa, y evitando en la medida de lo posible cualquier barrera digital que pueda interferir en la dimensión temporal de la praxis interpretativa. Esta actividad complementaria funciona como prueba final y como cierre de un proceso de aprendizaje online y colectivo. Asimismo, permite explorar ámbitos transversales y eminentemente prácticos abordados en otras asignaturas y de gran utilidad para el desarrollo profesional de los futuros egresados en educación musical superior. En este sentido, las titulaciones y los programas docentes siempre deben estar orientados a responder a las competencias exigidas por el mercado laboral (Infante-Moro, Infante-Moro y Gallardo Pérez, 2021: 147).

En cuanto al seguimiento y a la evaluación de la adquisición de las competencias planteadas en las asignaturas instrumentales conjuntas por parte del alumnado, se proponen diversas herramientas metodológicas. En primer lugar, las sesiones expositivas servirán para desarrollar los diferentes contenidos relativos a las unidades didácticas que compongan la asignatura. Este procedimiento, fácilmente realizable de manera online, servirá como instrumento para promover la participación activa del alumno a través de la realización de preguntas, permitiendo al tutor la detección de problemas y carencias a superar a partir de un trabajo específico y de su orientación en la búsqueda de fuentes complementarias. Este escenario de enriquecimiento bidireccional se puede alcanzar mediante la formulación de preguntas directas o indirectas, promoviendo el diálogo y el debate entre el alumnado, y contribuyendo a una absorción más completa de los conocimientos.

Debido a las particularidades de las asignaturas instrumentales conjuntas, la asistencia se convierte en una estrategia metodológica básica y en un requisito fundamental para la superación de las mismas. Esta es obligatoria y nunca podrá ser inferior al $80 \%$. En caso de que el alumno no alcance este porcentaje no será posible practicar una evaluación continua, viéndose obligado a realizar una prueba específica en la cual se incluyan todos los contenidos teórico-prácticos desarrollados 
SARA PÉREZ BORRAJO Y AARÓN PÉREZ-BORRAJO

EDUCACIÓN MUSICAL SUPERIOR Y PRÁCTICAS INSTRUMENTALES CONJUNTAS EN TIEMPOS DE COVID-19

en la materia. Por otro lado, recursos como seminarios o talleres también constituyen herramientas sumamente interesantes. En este tipo de eventos, un experto en un ámbito específico lo aborda en profundidad. Aquí, uno de los objetivos primordiales es el fomento de la participación del alumnado, ampliando así las posibilidades del tema y promoviendo su interconexión con otros aspectos no contemplados en un inicio. El hecho de que este tipo de actividades se planteen de manera no presencial y telemática favorece su realización no solo porque la disponibilidad del ponente aumenta, sino porque sus costes se reducen considerablemente.

Otra herramienta metodológica son las lecturas como forma de trabajo individual. Estas aportan un contenido complementario que favorece la consecución de los objetivos de la asignatura. Algunas de ellas pueden transformarse en estudios de caso. Es decir, a partir de la realidad que dichas fuentes presentan, los estudiantes buscan soluciones y respuestas mediante la aplicación de distintas perspectivas. Podríamos considerarlo como un tipo de aprendizaje a través de problemas, recorriendo diferentes caminos siempre bajo la orientación del tutor. Esta estrategia va inevitablemente ligada a otras, fácilmente materializables en un sistema no presencial, como las exposiciones y las presentaciones sobre contenidos relacionados con la materia. Por su parte, las tutorías, uno de los mecanismos más adecuados para seguir el progreso del alumno, sirven para responder a las inquietudes y a las dudas sobre las cuestiones abordadas. Estas permiten conocer las necesidades específicas del alumno, entre ellas las tecnológicas. Se convierten, por lo tanto, en una forma de sortear las brechas digitales y de trabajar por una participación plena del alumnado.

Al margen de la pertinencia de incluir una prueba final escrita u oral -ya sean preguntas a desarrollar, audiciones o análisis musical- en una asignatura instrumental conjunta, las grabaciones individuales, colectivas, sobre metrónomo y sobre pista a las que nos hemos referido en el proceso de enseñanza-aprendizaje anteriormente propuesto se convierten en objetos evaluables. En este sentido, Grande-Prado, García-Peñalvo, Corell Almuzara y Abellá-García (2021: 52) proponen un procedimiento de evaluación basado en la combinación de diferentes pruebas, apostando por la inclusión del alumnado y por una adaptación a sus particularidades, y haciendo hincapié en la protección de datos. Por supuesto, las actividades anteriormente mencionadas pueden ir acompañadas por otras de carácter complementario. Sin embargo, todas ellas están vinculadas al trabajo autónomo del estudiante, a la labor realizada por el alumno para reforzar su comprensión de los contenidos y al imperativo de cumplir con las tareas asignadas e indicadas por el profesor ${ }^{12}$.

12 Sobre este ámbito, aportaciones como las de González-González, Infante-Moro e Infante-Moro (2020) sirven para comprender los retos y las posibilidades que supone la implementación de un sistema de evaluación no presencial (E-Proctoring). Este procedimiento 
SARA PÉREZ BORRAJO Y AARÓN PÉREZ-BORRAJO

EDUCACIÓN MUSICAL SUPERIOR Y PRÁCTICAS INSTRUMENTALES CONJUNTAS EN TIEMPOS DE COVID-19

Con todo, teniendo en cuenta la sociedad plural y diversa ante la que nos encontramos, este modelo de educación online también debe contemplar medidas de atención a la diversidad del alumnado, promoviendo una relación inclusiva tanto entre los estudiantes como colectivo como entre estos y el profesorado. Esta cuestión demuestra que es posible crear contextos de igualdad a partir del conocimiento. $\mathrm{Al}$ respecto, contemplamos tres ámbitos: diversidad en función de la especialidad, diversidad en función del contexto sociocultural y diversidad en función de las capacidades y necesidades. Estas tres dimensiones permitirín la realización de una adaptación curricular siempre y cuando fuera preciso y debidamente justificado.

En cuanto a la diversidad del alumnado en función de su especialidad, aunque los contenidos y las actividades planteadas en las asignaturas instrumentales conjuntas sean iguales para todos, estos tratarán de aproximarse al ámbito de conocimiento del estudiante a través de la selección de los ejemplos, objetos de estudio, temas de los trabajos propuestos, etc. El cumplimento con las herramientas metodológicas anteriormente expuestas no impedirá una flexibilidad por parte del profesor, persiguiendo el máximo éxito en la adquisición de conocimientos del estudiante, independientemente de su especialidad.

Sobre la diversidad del alumnado en función de su contexto sociocultural, se incide en la rápida intervención del profesorado ante la identificación de conflictos debido a cuestiones extracurriculares y personales como el origen, las creencias religiosas, la identidad cultural, la identidad de género o la orientación sexual. Este tipo de actuaciones docentes e institucionales garantizan una educación y un desarrollo plenamente inclusivo. En relación con la diversidad del alumnado en función de sus capacidades y necesidades se debe tener en cuenta, si fuera el caso, la diversidad funcional de los alumnos, ya sea de tipo sensorial, motriz, cognitivo, etc. ${ }^{13}$. Atendiendo a esta, las actividades o los soportes a través de los cuales estas se realizan deberían ser adaptados. De idéntica forma, también se valorará la presencia de alumnado con altas capacidades, favoreciendo su integración y su colaboración en el desarrollo colectivo.

Asimismo, los alumnos participarán en la evaluación de la asignatura. No solo serán informados sobre los contenidos de las respectivas guías docentes y sus criterios de evaluación, sino que podrán conocer las calificaciones de cada una de las actividades evaluables en un plazo fijado previamente. Igualmente, se fomentará que las pruebas sean revisadas conjuntamente en el aula, indicando y proponiendo estrategias para la superación de los posibles errores. Recordemos que el alumno

permite supervisar y controlar de manera telemática la realización de pruebas evaluables por parte del alumno.

13 Sobre la relación entre música, educación musical y diversidad funcional son de interés aportaciones como las de Abramo (2012), Colwell (2002), Darrow (2003), Darrow (2015), Jellison y Taylor (2007) y Straus (2011). 
SARA PÉREZ BORRAJO Y AARÓN PÉREZ-BORRAJO

EDUCACIÓN MUSICAL SUPERIOR Y PRÁCTICAS INSTRUMENTALES CONJUNTAS EN TIEMPOS DE COVID-19

no solo tiene derecho a estar al tanto de los resultados de su proceso de aprendizaje, sino a ser evaluado conforme a criterios objetivos (garantías procedimentales en la evaluación). Por último, tras la finalización de la asignatura, al estudiante se le proporcionarán las indicaciones oportunas para acceder y cumplimentar un cuestionario online y anónimo sobre el desarrollo, contenido, interés y aprovechamiento de cada una de las unidades didácticas y de las diferentes actividades planteadas en ellas.

\section{CONCLUSIONES}

La situación de pandemia global originada por el COVID-19 supuso un gran reto, un estímulo, pero también un impulso necesario para la experimentación con sistemas educativos alternativos a los institucionalmente hegemónicos. Este nuevo escenario sociosanitario alteró de manera radical las variables y los parámetros que determinan nuestros comportamientos y modos de funcionamiento como individuos inmersos dentro de una compleja estructura social. El coronavirus propició situaciones y condiciones del todo imprevistas, forzando la adopción de estrategias como las que se plantean en este artículo. Desde nuestro punto de vista, la implantación de tales propuestas difícilmente hubiera sido exitosa dentro de un marco de «normalidad» o de continuum. No solo nos referimos a su rápida aceptación global, sino a que la situación de emergencia convirtió su pronta instauración en una necesidad.

Esta crisis sociosanitaria afectó, aunque de diferente modo, a todos los sistemas educativos a nivel global. Por ello, es de gran importancia realizar un profundo y exhaustivo análisis sobre la situación de la formación artística y, concretamente, sobre la enseñanza musical superior con el objetivo de optimizar su adaptación a esta nueva realidad. En este contexto debemos destacar las ventajas y las posibilidades que plantea la educación online, sin olvidar que, para que esta modalidad sea efectiva, es imprescindible la adquisición o la mejora de las habilidades TIC, tanto por parte del docente como del alumno. Así también, es preciso tener en consideración la denominada brecha digital, suprimiéndola a través de una firme respuesta institucional que garantice el acceso y la participación en la educación online en igualdad de condiciones.

El modelo metodológico que hemos desarrollado para las asignaturas instrumentales conjuntas se caracteriza por ser proactivo, cooperativo y colectivo. La acción promueve la intervención y el protagonismo del alumnado en su propio proceso de enseñanza-aprendizaje. En definitiva, esta propuesta está plenamente enfocada en el sujeto para el cual se diseña. Es decir, está fundamentada en el principio metodológico de atención personalizada y tiene en cuenta la proximidad y la flexibilidad de las actuaciones. Igualmente, debemos señalar el principio dialógico, 
SARA PÉREZ BORRAJO Y AARÓN PÉREZ-BORRAJO

EDUCACIÓN MUSICAL SUPERIOR Y PRÁCTICAS INSTRUMENTALES CONJUNTAS EN TIEMPOS DE COVID-19

resultado y consecuencia directa del diálogo activo basado en la argumentación coherente de las ideas expuestas.

Para su materialización, es importante disponer de recursos de calidad que satisfagan las necesidades de los estudiantes, entendiendo la calidad como un compromiso. Esta metodología trata de gestionar el conocimiento que produce el grupo en conjunto, actuando como una comunidad en la que todos los miembros son constructores y beneficiarios de dicha cooperación y donde el conocimiento colectivo contribuye a la mejora del propio aprendizaje individual. Los recursos metodológicos aquí empleados están orientados a favorecer la autoexpresión, la comunicación, la autonomía, la implicación, el compromiso, la motivación y el pleno desarrollo del alumno.

En este sentido, cabe destacar la importancia que las diferentes materias relativas a las prácticas instrumentales conjuntas tienen en el currículo del estudiante. Este tipo de asignaturas plantean grandes posibilidades a la hora de trabajar en una futura salida profesional y en una rápida inmersión laboral. Lo cierto es que, en la actualidad, los sistemas educativos se encuentran ante el reto de dar una respuesta satisfactoria a unas demandas que reflejan cuál es la sociedad en la que vivimos. Nos situamos en un mundo globalizado, muy competitivo, altamente cambiante y de una complejidad progresivamente en aumento, que exige profesionales -también en el sector musical-dispuestos a formarse de manera continua y con la capacidad de innovar y de adaptarse a nuevos escenarios.

\section{BIBLIOGRAFÍA}

Abramo, J. (2012). Disability in the Classroom: Current Trends and Impacts on Music Education. Music Educators Journal, 99(1), 39-45.

Bayley, J. G. y Waldron, J. (2020). «It's never too late»: adult students and music learning in one online and offline convergent community music school. International Journal of Music Education, 38(1), 36-51.

Brändström, S.; Wiklund, C. y Lundström, E. (2012). Developing distance music education in Arctic Scandinavia: electric guitar teaching and master classes. Music Education Research, 14(4), 448-456.

Brook, J. y Upitis, R. (2014). Can an online tool support contemporary independent music teaching and learning? Music Education Research, 17(1), 34-47.

Cabrera, L. (2020). Efectos del coronavirus en el sistema de enseñanza: aumenta la desigualdad de oportunidades educativas en España. Revista de Sociología de la Educación (RASE), 13(2), 114-139.

Cayari, C. (2018). Connecting music education and virtual performance practices from YouTube. Music Education Research, 20(3), 360-376.

Chao-Fernández, R.; Vázquez-Sánchez, R. y Felpeto-Guerrero, A. (2020). Audacity como herramienta para la creación de materiales educativos. Una aproximación a través 
SARA PÉREZ BORRAJO Y AARÓN PÉREZ-BORRAJO

EDUCACIÓN MUSICAL SUPERIOR Y PRÁCTICAS INSTRUMENTALES CONJUNTAS EN TIEMPOS DE COVID-19

del MOOC «Música para el siglo XXI». Revista Latinoamericana de Tecnología Educativa-RELATEC, 19(1), 121-137.

Colwell, C. M. (2002). Learning Disabilities in the Music Classroom: Implications for the Music Educator. Update: Applications of Research in Music Education, 21(2), 9-16.

Daniel, Sir J. (2020). Education and the COVID-19 pandemic. PROSPECTS, 49, 91-96.

Darrow, A.-A. (2003). Dealing with diversity: the inclusion of students with disabilities in music. Research Studies in Music Education, 21(1), 45-57.

Darrow, A.-A. (2015). Ableism and Social Justice: Rethinking Disability in Music Education. En C. Benedict et al. (coords.), The Oxford Handbook of Social Justice in Music Education (pp. 204-220). New York: Oxford University Press.

Daubney, A. y Fautley, M. (2020). Editorial Research: Music Education in a Time of Pandemic. British Journal of Music Education, 37(2), 107-114.

Decreto 171/2016, do 24 de novembro, polo que se establece o Plan de estudos das ensinanzas artísticas superiores de Música, nas especialidades de Dirección e Produción e Xestión, e se amplían os anexos do plan de estudos correspondente á especialidade de Interpretación, establecidos no Decreto 163/2015, do 29 de outubro, mediante a incorporación de novos itinerarios. Diario Oficial de Galicia, núm. 235, 12 de decembro de 2016, 54101-54170. Disponible en: https://0151cc31-c939-46cb-8f09f9239d6093df.filesusr.com/ugd/04f96b_2951ce8b598e4183a4065b1da4b96a3b.pdf

Gammeren, D. van y Szram, A. (2019). Threshold Concepts in Online Music Education: Transforming Conservatoire Training. En European Conference on e-Learning (pp. 588-592). Academic Conferences International Limited.

García-Peñalvo, F. J. (2020). Transformación digital en las universidades: implicaciones de la pandemia de la COVID-19. Education in the Knowledge Society, 22, e25465.

García-Peñalvo, F. J. y Corell, A. (2020). La COVID-19: ¿enzima de la transformación digital de la docencia o reflejo de una crisis metodológica y competencial en la educación superior? Campus Virtuales, 9(2), 83-98.

García-Peñalvo, F. J.; Corell, A.; Abellá-García, V. y Grande, M. (2020). La evaluación online en la educación superior en tiempos de la COVID-19. Education in the Knowledge Society, 21(12).

González-González, C. S.; Infante-Moro, A. e Infante-Moro, J. C. (2020). Implementation of E-Proctoring in Online Teaching: A Study about Motivational Factors. Sustainability, 12(8), 3488.

Grande-de-Prado, M.; García-Peñalvo, F. J.; Corell Almuzara, A. y Abella-García, V. (2021). Evaluación Superior durante la pandemia de la COVID-19. Campus Virtuales, 10(1), 49-58.

He, Y. (2020). Research on Online Teaching of Music Performance Based on Diversification and Intelligence. Take the Online Music Teaching during the COVID-19 as an Example. International Conference on E-Commerce and Internet Technology (pp. 193-196). Zhangjiajie: IEEE.

Infante, A.; Infante, J. C. y Gallardo, J. (2021). Análisis de las competencias digitales en el Máster de Turismo de la Universidad de Huelva. Campus Virtuales, 10(2), 141-151. 
SARA PÉREZ BORRAJO Y AARÓN PÉREZ-BORRAJO

EDUCACIÓN MUSICAL SUPERIOR Y PRÁCTICAS INSTRUMENTALES CONJUNTAS EN TIEMPOS DE COVID-19

Jellison, J. A. y Taylor, D. M. (2007). Attitudes toward Inclusion and Students with Disabilities: A Review of Three Decades of Music Research. Bulletin of the Council for Research in Music Education, 172, 9-23.

Kesendere, Y.; Sakin, A. Ş. y Acar, A. K. (2020). Educators' views on online/distance violin education at Covid-19 outbreak term. Journal for the Interdisciplinary Art and Education, 1(1), 1-19.

King, A.; Prior, H. y Waddington-Jones, C. (2019). Exploring teachers' and pupils' behaviour in online and face-to-face instrumental lessons. Music Education Research, 21(2), 197-209.

Koutsoupidou, Th. (2013). Online Distance Learning and Music Training: Benefits, Drawbacks and Challenges. The Journal of Open, Distance and e-Learning, 7(1), 143-152.

Kuric Kardelis, S.; Calderón Gómez, D. y Sanmartín Ortí, A. (2021). Educación y brecha digital en tiempos del COVID-19. Perfiles y problemáticas experimentadas por el alumnado para continuar sus estudios durante el confinamiento. Revista de Sociología de la Educación-RASE, 14(1), 63-84.

Leontiev, A. (1981). Actividad, conciencia y personalidad. La Habana: Editorial Pueblo y Educación.

Lloyd, M. (2020). Desigualdades educativas y la brecha digital en tiempos de COVID-19. En Educación y pandemia: una visión académica (pp. 115-121). Ciudad de México: Universidad Nacional Autónoma de México.

Orman, E. K. y Whitaker, J. A. (2010). Time Usage During Face-to-Face and Synchronous Distance Music Lessons. American Journal of Distance Education, 24(2), 92-103.

Rappoport, S.; Rodríguez, M. ${ }^{a}$ S. y Bressanello, M. ${ }^{a}$ (2020). Enseñar en tiempos de COVID-19: Una guía teórico-práctica para docentes. Montevideo: Organización de las Naciones Unidas para la Educación, la Ciencia y la Cultura.

Real Decreto 463/2020, de 14 de marzo, por el que se declara el estado de alarma para la gestión de la situación de crisis sanitaria ocasionada por el COVID-19. Boletín Oficial del Estado, núm. 67, 14 de marzo de 2020, 25390-25400.

Riley, P. E. (2009). Video-conferenced music teaching: challenges and progress. Music Education Research, 11(3), 365-375.

Rodicio-García, M. ${ }^{a}$ L.; Ríos-de-Deus, M. ${ }^{a}$ P.; Mosquera-González, M. ${ }^{a}$ J. y Penado Abilleira, M. ${ }^{a}$ (2020). La brecha digital en estudiantes españoles ante la crisis de la Covid-19. Revista Internacional de Educación para la Justicia Social, 9(3), 103-125.

Rujas, J. y Feito, R. (2021). La educación en tiempos de pandemia: una situación excepcional y cambiante. Revista Sociológica de la Educación (RASE), 14(1), 4-13.

Straus, J. N. (2011). Extraordinary Measures: Disability in Music. New York: Oxford University Press.

Vygotsky, L. (1972). El problema de la periodización por etapas del desarrollo del niño. Problemas de Psicología, 2, 114-123.

Waldron, J.; Mantie, R.; Partti, H. y Tobias, E. S. (2017). A brave new world: theory to practice in participatory culture and music learning and teaching. Music Education Research, 20(3), 289-304. 